\title{
Centrocestus formosanus em PEIXES DE ÁGUA DOCE destinados ao consumo humano em Minas Gerais, Brasil
}

\author{
Centrocestus formosanus in freshwater fish destined for \\ human consumption in Minas Gerais, Brazil
}

Pedro Henrique Magalhães Cardoso ${ }^{1}$

Avenida Prof. Dr. Orlando Marques de Paiva, 87, Butantã, São Paulo, SP, Brasil - CEP: 05508-270. E-mail: pedrohenriquemedvet@usp.br

RELVAS, R.S. ${ }^{2}$; BALIAN, S.C. ${ }^{3}$

\begin{abstract}
Como citar: RELVAS, Rachel Sordi; BALIAN, Simone de Carvalho; CARDOSO, Pedro Henrique Magalhães. Centrocestus formosanus em peixes de água doce destinados ao consumo humano em Minas Gerais, Brasil. Revista de Educação Continuada em Medicina Veterinária e Zootecnia do CRMV-SP, São Paulo, v.18, n.1, 2020. Doi.10.36440/recmvz.v18i1.38029

Cite as: RELVAS, Rachel Sordi; BALIAN, Simone de Carvalho; CARDOSO, Pedro Henrique Magalhães. Centrocestus formosanus in freshwater fish destined for human consumption in Minas Gerais, Brazil. Journal of Continuing Education in Animal Science of CRMV-SP, São Paulo, v.18, n.1, 2020. Doi.10.36440/recmvz.v18i1.38029
\end{abstract}

\section{Resumo}

Centrocestus formosanus infesta as brânquias dos peixes, causando altas morbidades e mortalidades e, por consequência perdas econômicas. Este estudo relata a identificação de metacercárias de $C$. formosanus em peixes de água doce destinados ao consumo humano, provenientes de uma piscicultura em Minas Gerais. Um total de 172 peixes juvenis foram examinados e, destes, 107 apresentavam metacercárias. Esta ocorrência é indicativa de falhas nas práticas de controle de caramujos e representa risco à Saúde Pública.

Palavras-chave: Centrocestus. Metacercárias. Aquicultura. Saúde animal. Saúde pública.

\section{Abstract}

Centrocestus formosanus infects fish gills, producing high morbidities and mortalities and, consequently, economic losses. This study reports the identification of metacercaries of $C$. formosanus on fishes destined for human consumption, from a fish farming at Minas Gerais. From 172 juvenile fishes examinated 107 presented metacercaries in their gills. Its presence suggests lack of good management practices regarding snail control and it is a Public Health risk.

Keywords: Centrocestus. Metacercaries. Aquaculture. Animal health. Public health.

1 Médico-veterinário PhD

2 Médica-veterinária graduada pela Faculdade de Medicina Veterinária e Zootecnia, Universidade de São Paulo, São Paulo, SP, Brasil

3 Professora livre docente da Faculdade de Medicina Veterinária e Zootecnia, Universidade de São Paulo, São Paulo, SP, Brasil 


\section{Introdução}

A produção em terra firme com tanques escavados destaca-se como a forma mais utilizada mundialmente para a criação de peixes na aquicultura. Todavia, o cultivo intensivo interfere na relação hospedeiro-parasito, principalmente em função do estresse, provocando imunossupressão nos peixes que se tornam mais suscetíveis a infestações parasitárias, em virtude do desequilíbrio da tríade hospedeiro-ambiente-agente (JERÔNIMO, 2013).

Centrocestus formosanus (espécie digenética da classe Trematoda, do filo Platelminto) é originário da Ásia e parasita, sob a forma de metacercárias, principalmente brânquias de peixes. Está mundialmente distribuído, com relatos em países de diferentes continentes: Estados Unidos, (WOO, 2006), Peru (PULIDO-MURILLO et al., 2018), Egito (YOUSIF et al., 2016), Croácia (GJURČEVIĆ et al., 2007) e Austrália (EVANS; LESTER, 2001). Diferentemente de outros trematoides parasitas de peixes, C. formosanus pode causar altas morbidades e mortalidades, acarretando perdas econômicas significativas, especialmente em fases larvais ou juvenis (WO0, 2006; NOGA, 2010). É importante salientar que este parasita tem potencial zoonótico e oferece risco à saúde humana quando da ingestão de peixes crus ou mal-cozidos infestados com metacercárias. Ao ingerir as metacercárias vivas (fase larval), o ser humano comporta-se como hospedeiro definitivo, desenvolvendo no intestino a forma adulta (DE; LE, 2011; CHAl et al., 2013; NOGA, 2010).

o Centrocestus formosanus necessita de um hospedeiro intermediário para completar o seu ciclo de vida, o caramujo aquático Melanoides tuberculatus (NOGA, 2010). Esse molusco, foi registrado no Brasil na década de 1960 de desde então foi se distribuindo gradativamente pelos estados brasileiros e, na atualidade, é considerado como uma população bem estabelecida (W00, 2006; PINTO; MELO, 2010).

O presente trabalho relata o registro da presença de metacercárias encapsuladas de $C$. formosanus em peixes de água doce destinados ao consumo humano, provenientes de uma piscicultura localizada na região da Zona da Mata, estado de Minas Gerais, Brasil .

\section{Descrição do caso}

Um produtor de peixes de produção, localizado na região da Zona da Mata Mineira, relatou que sempre, durante a rotina de transferência dos alevinos de carpas coloridas para os tanques escavados para engorda, percebia que muitos animais sumiam. 0 problema era maior com carpas coloridas do que com outros peixes produzidos. Suspeitando que a ocorrência fosse consequência de alguma doença de etiologia parasitária, no período compreendido entre dezembro de 2018 a maio de 2019, foram capturados 172 peixes juvenis. Os peixes foram transportados vivos em embalagens plásticas com oxigênio até uma distribuidora de peixes ornamentais localizada na Grande São Paulo, onde foram realizados os exames. A execução da investigação foi autorizada pela CEUA/FMVZ (CEUA no 1976201218).

Os peixes foram anestesiados com Eugenol (75 mg/L) (ROUBACH et al., 2005), e eutanasiados por secção da medula espinhal (NOGA, 2010). Após necropsia e visualização de todos os arcos branquiais sob microscópio óptico (montadas entre lâmina e lamínula), foram identificados 107 (62\%) peixes infestados por metacercárias de C. formosanus: 12 de 72 tilápiasdo-Nilo (Oreochromis niloticus); 19 de 19 carpas coloridas (Cyprinus carpio haematopterus); 23 de 23 bagres americanos/catfish (Ictalurus punctatus); 17 de 17 matrinxãs (Brycon cephalus); 21 de 22 carpas capim (Ctenopharyngodon idella) e 15 de 19 lambaris (Astyanax bimaculatus). A Tabela 1 apresenta a frequência de ocorrência, a intensidade média e a abundância média para cada espécie e para o total de animais amostrados. 
Tabela 1. Presença de Centrocestus formosanus nas guelras de peixes de água doce, capturados em um criatório localizado no estado de Minas Gerais, Brasil, segundo o tipo de peixe, a frequência de ocorrência, a intensidade média e a abundância, para cada espécie e para o total de animais examinados. Capturas efetuadas no período de dezembro de 2018 a maio de 2019

\begin{tabular}{|c|c|c|c|c|c|c|c}
\hline \multicolumn{2}{|c|}{$\begin{array}{c}\text { Tilápia-do- } \\
\text { nilo }\end{array}$} & $\begin{array}{c}\text { Carpa } \\
\text { colorida }\end{array}$ & $\begin{array}{c}\text { Bagre } \\
\text { americano }\end{array}$ & Matrinxã & Carpa capim & Lambari & Total \\
\hline$P$ & 0,17 & 1,00 & 1,00 & 1,00 & 0,95 & 0,79 & 0,62 \\
\hline I & 2,08 & 250,95 & 530,57 & 63,29 & 93,67 & 5,73 & 188,08 \\
\hline A & 0,35 & 250,95 & 530,57 & 63.29 & 89,41 & 4,53 & 117,01 \\
\hline
\end{tabular}

Fonte: Autoria própria (2020).

Legenda 1. Frequência de ocorrência (P), Intensidade média (I) e Abundância média (A) de infestação

O exame microscópico revelou a presença de cistos arredondados (Figura 1a) a ovalados no tecido dos filamentos branquiais, contendo em seu interior uma estrutura em forma de ' $X$ ' (Figura 1b). Em algumas brânquias selecionadas para análise histopatológica (coloração por hematoxilina e eosina) foi constatada a intensa proliferação de tecido cartilaginoso formando uma cápsula ao redor dos cistos (Figura 1c), características patognomônicas de infestação por C. Formosanus (WOO, 2006; NOGA, 2010).

Figura 1. Cistos arredondados a ovalados [objetiva 4x] (a); com estrutura em forma de ' $X$ ' (b) [objetiva 20x] e intensa proliferação de tecido cartilaginoso formando uma cápsula ao redor dos cistos [H.E.] (c) em brânquias, que em altas infestações deslocam o opérculo, cranialmente, deixando-as expostas e visíveis em Cyprinus carpio (d); caramujos (e); aves nos tanques escavados de criação (f)
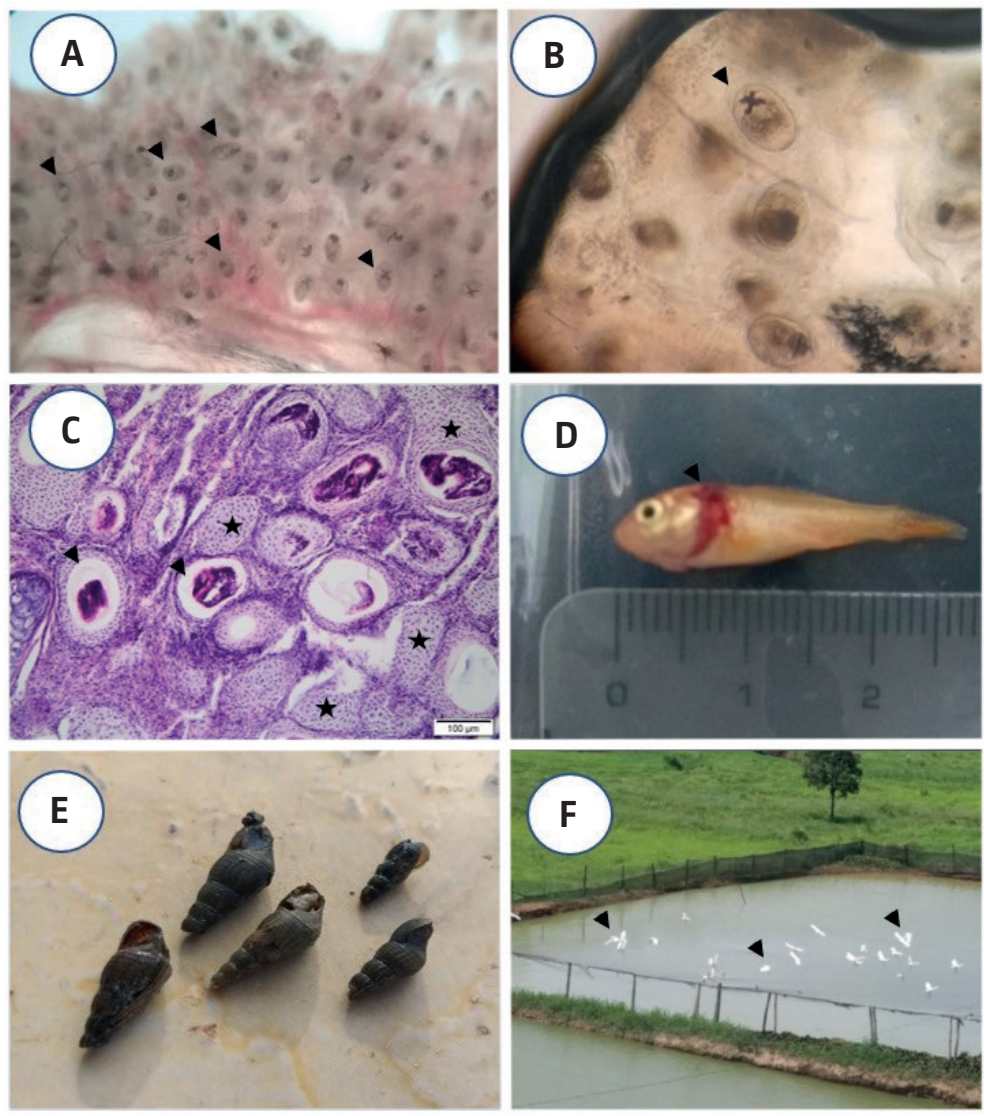

Fonte: Relvas (2020). 
Em 29\% (31/107) dos animais foi constatada a presença de movimentos operculares acelerados e brânquias macroscopicamente aumentadas de volume. Tal aumento em alguns indivíduos, provocava o deslocamento cranial do opérculo que ficaram expostos e visíveis (Figura 1d). Em uma visita técnica realizada na propriedade de criação dos peixes foi confirmada a presença de caramujos (Figura 1e) e de aves nas imediações dos tanques escavados (Figura 1f).

\section{Discussão e Conclusão}

O ciclo de vida do C. formosanus depende de três hospedeiros para se completar: dois hospedeiros intermediários (HI) e um definitivo (HD). 0 molusco M. tuberculatus atua como 10 HI. Em forma de cercária, o parasita sai para a água em busca do $20 \mathrm{HI}$ (peixes) e se transforma em metacercária ao invadir as brânquias. Aves e mamíferos piscívoros se alimentam do peixe infestado e completam o ciclo, permitindo o desenvolvimento da fase adulta (HD) (NOGA, 2010). Peixes com intensas cargas parasitárias também apresentam metacercárias nas nadadeiras, na cabeça e em outras áreas do corpo (SUMUDUNI et al., 2017).

A espécie $C$. formosanus é de extrema importância para pisciculturas em contato com aves e caramujos, provocando alta morbidade e mortalidade. Isso se deve ao severo dano causado nas brânquias (NOGA, 2010). Peixes juvenis e imunossuprimidos são mais suscetíveis (W00, 2006; SUMUDUNI et al., 2017). WOO, 2006 relatou que I. punctatus foi facilmente infestado quando exposto experimentalmente ao C.formosanus (W00, 2006). Esse fenômeno pode explicar 0 fato da maioria dos peixes examinados possuírem elevada quantidade de metacercárias nas brânquias, com exceção da tilápia (Oreochromis niloticus), na qual a intensidade do parasitismos foi menor .

Nos animais parasitados foi observada a existência de uma resposta tecidual exacerbada nas brânquias, com proliferação do tecido cartilaginoso, aumento dos filamentos branquiais, evoluindo para a sua fusão (perda da morfologia normal) e perda funcional da capacidade de troca gasosa, levando a baixa eficiência respiratória. Macroscopicamente, foi constatada a hipertrofia das brânquias e o opérculo deslocado anteriormente o que corrobora com os registros de (SCHOLZ; SALGADO-MALDONADO, 2000; WOO, 2006; NOGA, 2010).

No Brasil, desde o início dos anos 2000 já havia sido registrado o transporte de metacercárias pelo do M. tuberculatus, porém, a espécie transportada não havia sido identificada. Foi em 2010, em Minas Gerais, que a espécie da metacercária foi identificada como C. formosanus (PINTO; MELO, 2010).

A forma encistada da metacercária dificulta o tratamento da infestação, pois sua conformação impede que o medicamento chegue à larva. Dos medicamentos que podem ser empregados para o tratamento dos peixes parasitados pelo C.formosanus, o praziquantel é o mais eficaz. A ivermectina não possui alta eficácia e o triclorfon é ineficaz. Entretanto, mesmo mortas, as metacercárias continuam presentes no tecido, podendo liberar toxinas e impossibilitando a regeneração do tecido danificado e a recuperação completa do animal (W00, 2006).

A prevenção é a medida de controle mais importante para evitar os prejuízos causados pela infestação dos peixes pelo C. formosanus. A melhor estratégia é o controle dos hospedeiros intermediários e evitar o contato com os hospedeiros definitivos. Deve-se impedir o contato entre peixes e aves, impedindo que elas se alimentarem de peixes e/ou de suas carcaças. 0 M. tuberculatus é relativamente resistente a moluscidas e desinfetantes (NOGA, 2010). Portanto, o combate ao C.formosanus assenta-se na aplicação de um conjunto de procedimentos: uso de herbicidas, controle do ambiente (remoção constante da vegetação e das algas dos tanques), cal, vazio sanitário, secagem dos tanques entre lotes e controle biológico (introdução de peixes predadores de moluscos) (WO0, 2006; SUMUDUNI et al., 2017). 
A maioria das espécies de peixes examinadas no presente caso, possuíam elevada concentração de metacercárias de C. formosanus e se encontravam na fase juvenil, isto é, na fase altamente suscetível a infestações. Essa constatação indica a existência de um grave erro no manejo de controle do caramujo somada à má prática da possibilidade do estabelecimento de contato entre peixes e aves selvagens.

Considerando a produção de peixes destinados ao consumo humano e que os trematoides digenéticos, no geral, possuem potencial zoonótico (LUQUE, 2004; WO0, 2006; NOGA, 2010) se os seres humanos ingerirem as metacercárias do C. formosanus, vivas haverá um elevado risco de que a forma adulta do parasita venha a se desenvolver no intestino do consumidor. Frequentemente os peixes infestados pelo C. formosanus apresentam altas concentrações do parasita nas brânquias, porém eles também podem estar presente em outras regiões do animal como o corpo e cabeça (SUMUDUNI et al., 2017), aumentando o risco da infestação dos seres humanos. Apesar de ser necessário ingerir a cabeça, as brânquias ou a pele de animal (cru ou com temperatura e/ou tempo de cocção insuficientes) para contrair o parasita, já existem indícios do potencial zoonótico deste parasita. Entretanto, há poucos estudos sobre manifestações clínicas e prevalência da infestação em humanos no mundo e no Brasil, a maioria das investigações disponíveis foram realizadas na Ásia (DE; LE, 2011; CHAl et al., 2013).

De e Le (2011), no Vietnã, estudando seres humanos com resultado positivo para a presença de ovos de trematoides nas fezes, escolheram 10 para identificar a espécie. Das dez amostras, três revelaram a presença de $C$. formosanus concomitantemente com outras espécies. Chai et al. (2013) também reportaram, em Laos, infestações mistas de $C$. formosanus com outros trematoides em sete indivíduos. Tais pacientes relatavam dor epigástrica em graus variados, indigestão e diarreia ocasional. Contudo, não se pôde afirmar a correlação dos sintomas com a espécie alvo deste estudo, devido à presença concomitante de outros parasitas. Em ambos os casos, as pessoas possuíam o hábito de consumir peixe cru ou mal cozido.

Os achados neste estudo permitem concluir que: 1) metacercárias encapsuladas de C. formosanus ocorrem em peixes de água doce produzidos na Zona da Mata, estado de Minas Gerais, Brasil e o consumo desses peixes mal cozidos ou crus representa risco para o consumidor; 2) há deficiências nas boas práticas de manejo em piscicultura de peixes para consumo humano na Zona da Mata de Minas Gerais, com relação ao controle de C.formosanus e 3) é possível evitar a ocorrência de metacercárias de C.formosanus em peixes destinados ao consumo humano a partir da adoção de rigorosas práticas de controle do molusco e ações que impeçam o contato entre peixes com aves selvagens (CHAl et al., 2013). 


\section{Referências}

CHAl, J. Y. et al. Centrocestus formosanus (Heterophyidae): human infections and infection source in Lao PDR. Journal of Parasitology, v. 99, n. 3, p. 531-536, 2013. Disponível em: http://dx.doi. org/10.1645/12-37.1. Acesso em: 28 jul. 2019.

$D E, N$. V.; LE, T. H. Human infections of fish-borne trematodes in Vietnam: prevalence and molecular specific identification at an endemic commune in Nan Dinh province. Experimental Parasitology, v. 129, n. 4, p. 355-361, 2011. Disponível em: https://doi.org/10.1016/j.exppara.2011.09.005. Acesso em: 28 jul. 2019.

EVANS, B. B.; LESTER, R. J. G. Parasites of ornamental fish imported into Australia. Bulletin of the European Association of Fish Pathologists, v. 21, n. 2, p. 51-55, 2001. Disponível em: https://www. researchgate.net/publication/46055603_Parasites_of_ornamental_fish_imported_into_Australia. Acesso em: 3 ago. 2019.

GJURČEVIĆ, E. et al. Metacercariae of Centrocestus formosanus in goldfish (Carassius auratus L.) imported into Croatia. Helminthologia, v. 44, n. 4, p. 214-216, 2007. Disponível em: https://doi.org/10.2478/ s11687-007-0034-4. Acesso em: 3 ago. 2019.

JERÔNIMO, G. T. et al. Métodos para coleta de parasitos de peixes. Macapá: Embrapa, 2011. (Circular Técnica, 39).

NOGA, E. J. Fish disease: diagnosis and treatment. 2. ed. Singapore: Wiley-Blackwell, 2010.

PINTO, H. A.; MELO, A. L. Melanoides tuberculata (Mollusca: Thiaridae) as an intermediate host of Centrocestus formosanus (Trematoda: Heterophyidae) in Brazil. Revista do Instituto de Medicina Tropicação de São Paulo, v. 52, n. 4, p. 207-210, 2010. Disponível em: http://www.scielo.br/scielo. php?script=sci_arttext\&pid=S0036-46652010000400008\&lng=en\&nrm=isso. Acesso em: 25 jul. 2019.

PULIDO-MURILLO, E. A. et al. Fishborne zoonotic trematodes transmitted by Melanoides tuberculate snails, Peru. Emerging Infectious Disease, v. 24, n. 3, p. 606-608, 2018. Disponível em: https://doi. org/10.3201/eid2403.172056. Acesso em: 3 ago. 2019.

ROUBACH, R. et al. Eugenol as an efficacious anaesthetic for tambaqui, Colossoma macropomum (Cuvier). Aquaculture Research, v. 36, n. 11, p. 1056-1061, 2005. Disponível em: https://doi.org/10.1111/ j.1365-2109.2005.01319.x. Acesso em: 5 ago. 2019.

SCHOLZ, T.; SALGADO-MALDONADO, G. The introduction and dispersal of Centrocestus formosanus (Nishigori, 1924) (Digenea: Heterophyidae) in Mexico: a review. American Midland Naturalist, v. 143, n. 1, p. 185-200, 2000. Disponível em: http://www.bioone.org/doi/full/10.1674/00030031\%282000\%29143\%5B0185\%3ATIADOC\%5D2.0.CO\%3B2. Acesso em: 25 jul. 2019.

SUMUDUNI, B. G. D.; MUNASINGHE, D. H. N.; ARULKANTHAN, A. Survivability and transmission rate of Centrocestus sp. cercariae larvae to early fingerlings of Koi carp (Cyprinus carpio) and lethal number of parasites to fry stage with site preference of infection. Journal of Fisheries and Life Sciences, v. 2, n. 2, p. 35-39, 2017. Disponível em: http://www.fishlifesciencejournal.com/archives/2017/2/2/ DECEMBER/34. Acesso em: 25 jul. 2019.

YOUSIF, F. et al. The first record of Centrocestus formosanus (Nishigori, 1924) (Digenea: Heterophyidae) in Egypt. Experimental Parasitology, v. 168, p. 56-61, 2016. Disponível em: https://doi.org/10.1016/j. exppara.2016.06.007. Acesso em: 3 ago. 2019.

WOO, P. T. K. (Ed.). Fish diseases and disorders. 2. ed. King's Lynn: CABI, 2006. 